\title{
Integrated Method of Reducing the Threat of Endogenous Fires in Hard Coal Mines
}

\author{
Dawid Szurgacz ${ }^{1 *}$, Leszek Sobik ${ }^{2}$, and Jarosław Brodny ${ }^{3}$ \\ ${ }^{1}$ Polska Grupa Górnicza S.A., ul. Powstańców 30, 40-039 Katowice, Poland \\ ${ }^{2}$ KWK ROW ruch Chwałowice, ul. Przewozowa 4, 44-206 Rybnik, Poland \\ ${ }^{3}$ Silesian University of Technology, Faculty of Organization and Management, Roosevelta 26, 41-800 \\ Zabrze, Poland
}

\begin{abstract}
Underground exploitation of hard coal based on a longwall system is increasingly exposed to various types of threats. In particular, this applies to endogenous fire threat. It results from coal left in the goaf, which due to favourable climatic conditions may lead to self-heating and self-ignition. In practice, due to the maintenance of the roof or the passage through the fault zones, coal is often left in the caving zone, which significantly increases the possibility of endogenous fires in this zone. Therefore, it is necessary to develop an effective prevention solution. The method presented in this paper combines all previously used methods aimed at reducing fire hazard. The levels of fire hazard indicators significantly dropped in the areas where the method had been applied. This, in turn, enabled safe mining operation in the tested longwalls, their decommission and the isolation of longwall workings. The results confirm the validity of the adopted assumptions. The method is based on practical and effective use of preventative measures designed to reduce endogenous fire threat. It can be successfully applied not only in mines without extensive fire prevention system but also in mines where other prevention methods are successfully used. It may support and supplement them. The article discusses basic assumptions of the method and presents a model of application.
\end{abstract}

\section{Introduction}

World underground mining of hard coal is carried out in various mining and geological conditions. In most cases, these conditions lead to various natural hazards during the mining process. Apart from the methane hazard $[1,2,4,8,11,14,16]$, the most dangerous risks include fire hazard $[5,13,15]$. Underground fires in mines are divided into exogenous and endogenous ones. In both cases, these phenomena are very dangerous. They may lead to serious consequences due to the spread of combustion products in the closed area of mining excavations. Gases and fumes rapidly spreading in the mine workings are extremely dangerous to the safety and life of the miners working underground. In many cases, it is also necessary to exclude entire mine areas due to fire hazard. In particular, this applies to endogenous fires, which most often start in the goafs or inactive excavations [7, 15]. They are most often recorded on the basis of effects, i.e. an increased amount of carbon monoxide in the ventilation air. Unfortunately, in contrast to surface conditions, the

\footnotetext{
*Corresponding author: dawidszurgacz@,vp.pl
} 
combustion products are very difficult to dilute in the ventilation air, which creates an additional hazard for the miners $[5,10]$.

Endogenous fires also result in substantial economic losses as it is very difficult to locate them (e.g. in goafs). All exploitation areas are usually isolated in order to cut off the oxygen supply and the operation may be resumed only after the fire stops by itself. Such a process is associated with large losses for mining enterprises and very often a long waiting time.

In the case of endogenous fires, their main cause is the carbon leftover in the goaf. Coal in the goafs is usually caused by off-balance deposits located in the roof, or as a result of geological disturbances in the longwall. Sometimes, due to the very weak floor rocks, there is a need to leave a subterranean layer of coal (the so-called patching) to prevent the against roof fall $[13,15]$. These factors cause endogenous fires to be inextricably linked to the exploitation of coal.

Underground exploitation due to the resources of coal and its significance for the global energy sector will be continued and probably with increasing intensity. This is evidenced by global coal resources shown in Figure 1 [9].

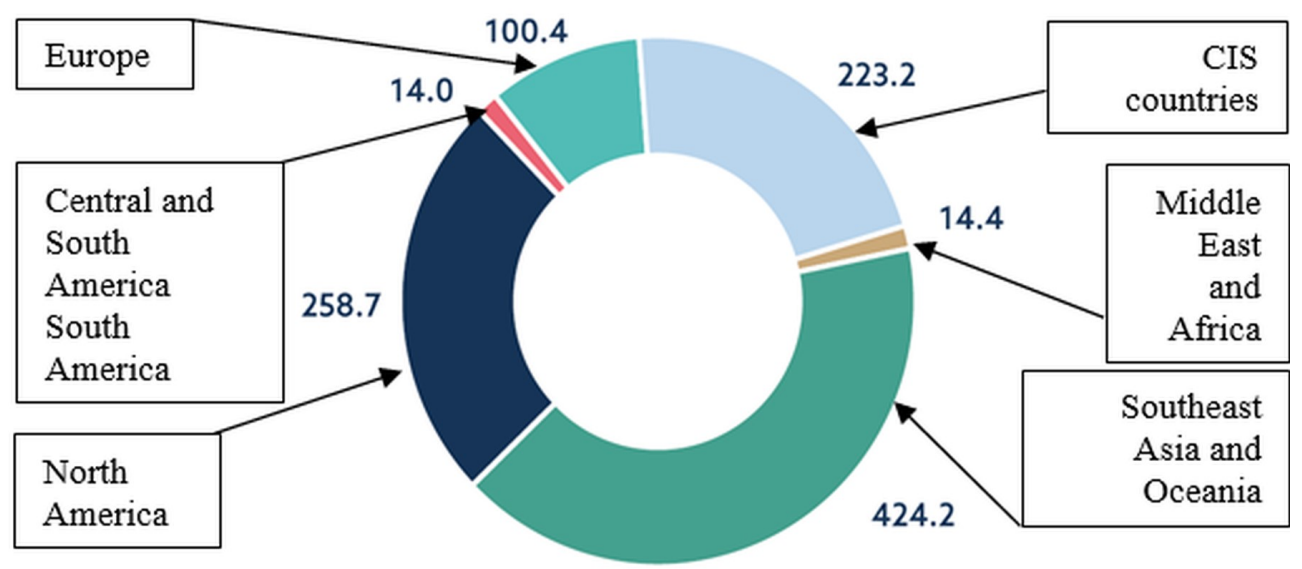

Fig. 1. Global resources of fossil fuels at the end of 2017 in billion $\mathrm{Mg}$ [9].

The global economy in many countries is to a large extent based on coal, which is still one of the cheapest sources of energy.

It is necessary to undertake actions that are to limit the negative effects of events resulting from these threats in order to minimize the effects of various natural hazards, including fire hazards.

In the case of endogenous fire threats, actions are taken, which are to limit the amount of coal left in the goaf and to prevent it.

These activities may only become effective if its foundation includes practical experience and scientific analysis. The paper presents an example of the application of a new method of reducing the threat of endogenous fires in hard coal mines. This method involves the simultaneous use of many solutions that have been used separately so far. This approach also results from very difficult and the specific conditions under which the operation was carried out in the studied area. The article discusses the developed method and presents the selected results of its practical application. 


\section{Characteristics of the method}

Chwałowice mine in Poland is characterised by high fire risk. Each longwall where coal is planned to $b$ left in goafs is fed with pipelines for supplying ash and water mixtures and inert gases. These pipelines are built in longwall walkways and outlets located from $60 \mathrm{~m}$ to $100 \mathrm{~m}$ behind the longwall face. Depending on the shape of the fire hazard, one to three main pipelines for conducting fire prevention are carried out in the longwall walkways. The construction in the caving zone of these pipelines is the foundation of the success of preventive actions based on the supply of ash-water mixtures and inert gases.

The ventilation system in caving longwalls in this area is based on $U$ method and therefore an integrated method of eliminating the fire hazard was developed and applied there. This method is based on feeding an ash-water mixture or an ash-water mixture with a lucrative gas, in this case carbon dioxide. It is also possible to supply inert gas itself (carbon dioxide) from the top gate (ventilation), which discharges the used air. The second inert gas (in this case nitrogen) is given from the side of the bottom gate that supplies fresh air.

The nitrogen (as an inert gas) fed to the goafs from the side of the bottom gate, according to the distribution of aerodynamic potentials, is intended to limit the migration of air to the caving zone. In accordance with the assumptions of the adopted prevention measures, the administration of inert gas (nitrogen) to goafs primarily decreases the oxygen concentration in the goaf, which significantly limits the development of self-heating processes. Inertisation with the nitrogen of the caving zone also does not allow the formation of an explosive mixture, especially in methane-contaminated seams.

The second element of the developed method is simultaneous feeding of ash-water mixtures from the side of the top gate. The use of mixtures of fly ash mainly causes caulking, cracks and fissures in the rock mass to seal, limiting the possibility of migration of oxygen-containing air to the goafs. It should be remembered that goafs are a highly porous medium through which, depending on its permeability, air and gas mixtures can flow, which promotes the emergence of ventilation hazards $[1,3,5,17]$.

The ash-water mixture which has good penetration parameters and so that it penetrates deeply into the goafs by caulking it and thus reducing the increase of the potential fire hazard.

Another significant and one of the most effective factors confirmed by many years of experience is the feeding of a second inert gas (carbon dioxide) from the top gate. The carbon dioxide moving to the zone of the caving zone should hinder the development of coal self-heating by reducing the rate of coal oxidation in the goaf. Carbon dioxide, due to the very intense bonding of sorbent forces with coal and rocks, covers the coal crumbled in the incineration, thus limiting the access to oxygen.

An important step is to achieve appropriate parameters of air flow, and thus aerodynamic potentials around the goafs $[6,7,12]$. The selection of the parameters of this flow allows to reduce the decreases in the potentials affecting the goaf, which allows to maintain an atmosphere that hinders the oxidation of carbon (this is the so-called neutral/inert atmosphere).

Several years of experience prove that the greatest effects in carbonation inertisation are obtained when the carbon dioxide feed will be carried out in the initial phase of the selfheating process of coal. It is therefore very important to conduct early detection of fires, and air quality tests (chromatographic analyses) play an especially important role. Increased carbon monoxide values as well as increase in the value of hydrocarbons mainly such as ethylene, propylene, acetylene or hydrogen in the initial phase of the coal self-heating 
process allow for early identification of the fire hazard, which enables initiation and selection of appropriately intensified and integrated preventive measures. This in turn allows quick start of intensified preventive actions enabling safe exploitation of longwalls, and then their recommissioning.

The monitoring of the quantity of injected gases and the analysis of aerodynamic potential shaping are usually carried out on the basis of current results of precise chromatographic analyses of gases taken mainly from the caving zone of the top gate. They are later used to determine changes in the temperature of heated coal.

In the case of limited caulking capacity (longitudinal longwalls) and low operational progress, the use of goaf inertisation with carbon dioxide and nitrogen is the most effective factor to stop the development of self-heating of coal in the goaf.

As a supporting factor in the presented methodology of limiting the fire hazard, besides caulking with ash-water mixtures and inertisation with inert gases (carbon dioxide and nitrogen), anti-pyrogenic foams are used. These foams, also called inhibitors, are substances that directly affect the coal, which limit or eliminate the oxidation process. The idea of the presented method, including all the elements discussed, is shown in Figure 2.

In the case of the tested mine, the device for producing anti-pyrogenic foam (foamed SIG mining inhibitor) is usually mounted in the top gate, and the generated foam is injected into goafs at places where coal is left. This foam, in addition to limiting the chemical activity of coal, also creates screens isolating the goaf space from the working space further reducing the air supply to goafs.

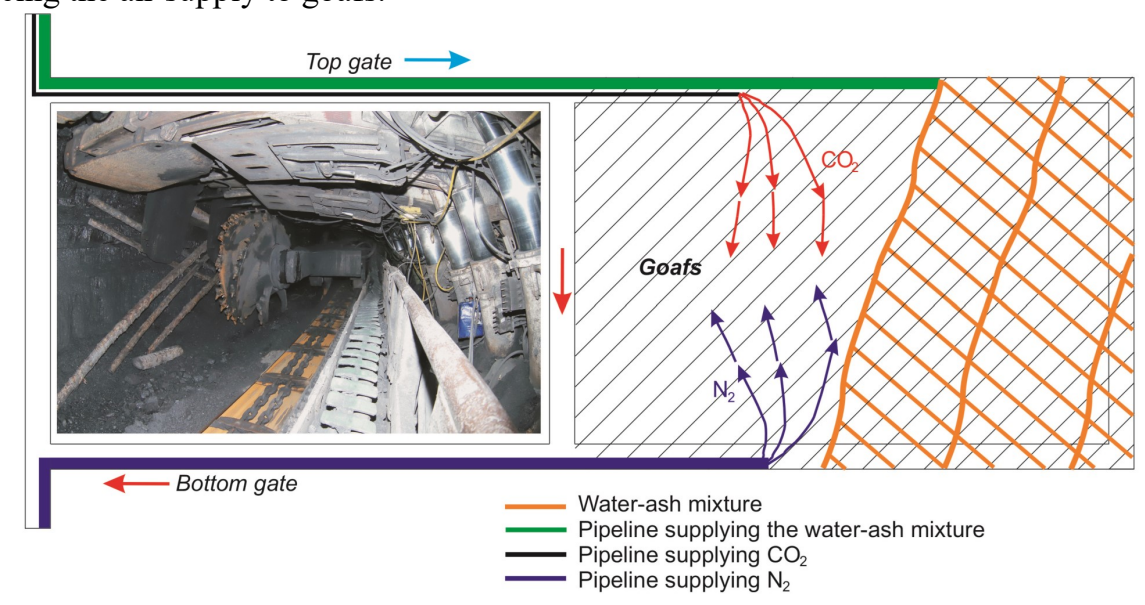

Fig. 2. An integrated method to reduce the fire hazard in the area of mining exploitation

\section{Results}

The presented method of limiting the threat of endogenous fires in hard coal mines is based on the combination of various prevention measures. It also uses various physicochemical properties of individual inert gases, anti-pyrogenic foams and ash-water mixtures in relation to mine air, rocks and coal. In particular, this applies to goafs, an area in which endogenous fires most often occur.

Carbon dioxide is heavier than air in the lower part of the space of inertised goafs, and nitrogen as lighter than air fills the remaining space destined for inertised of goafs. As a result of this method, both gases fill the space of inertised goafs. Using the advanced regulation of aerodynamic potentials, it is possible to trigger the intended motion of this 
mixture of inert gases in the space of inertised goafs. When applying caulking to ash and water mixtures and administering anti-pyrogenic foams, a high efficiency of these activities in reducing the occurrence of endogenous fire is obtained.

The developed method was used underground in several longwalls. The results are very good. In particular, this applies to longwalls with a very high tendency of coal to self-ignite with a simultaneous short-term fire incubation. Its application enabled safe operation and recommissioning.

This is confirmed by the results of changes in the content of carbon monoxide (CO) and unsaturated hydrocarbons of the $\mathrm{C}_{2}-\mathrm{C}_{4}$ group during operation and recommissioning of one of these longwalls, which are shown in Figures 3, 4 and 5.

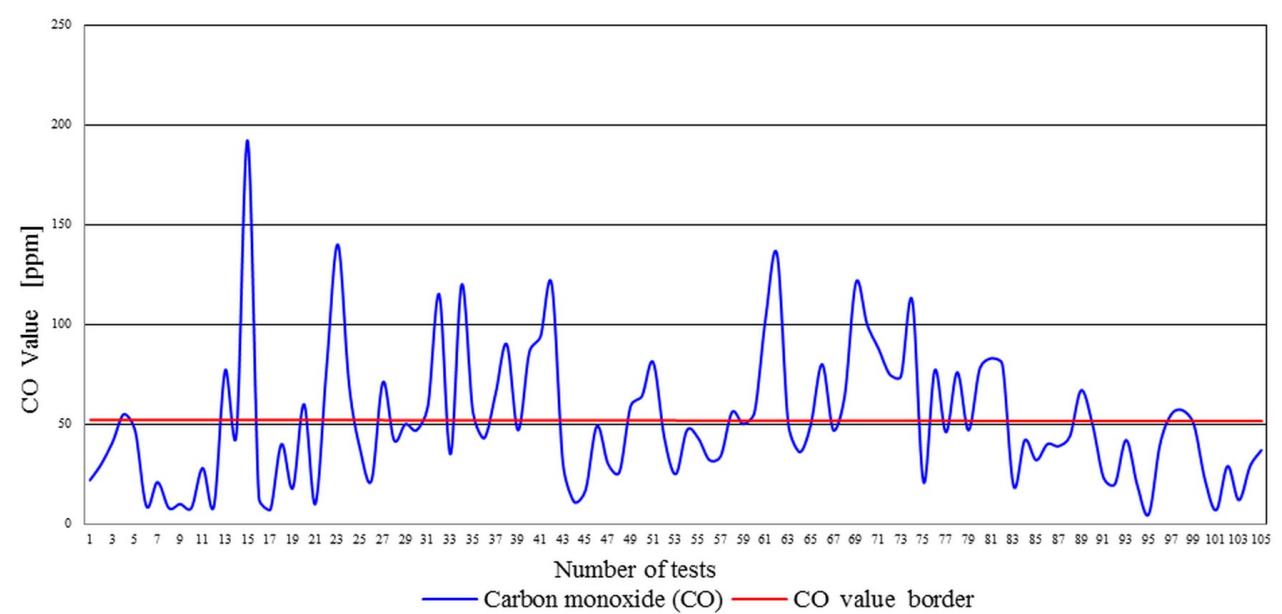

Fig. 3. Carbon monoxide concentrations in gas samples taken from goafs of an active longwall using an integrated method to reduce the risk of endogenous fires.

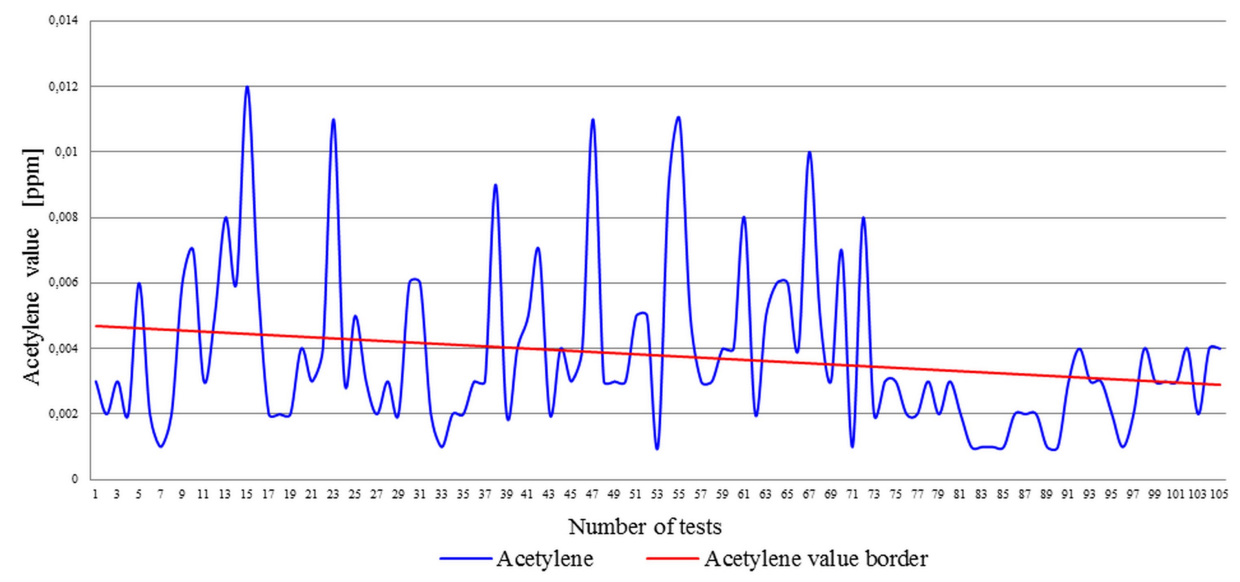

Fig. 4. Acetylene concentration in gas samples taken from goafs of an active longwall using an integrated method to reduce the risk of endogenous fires. 


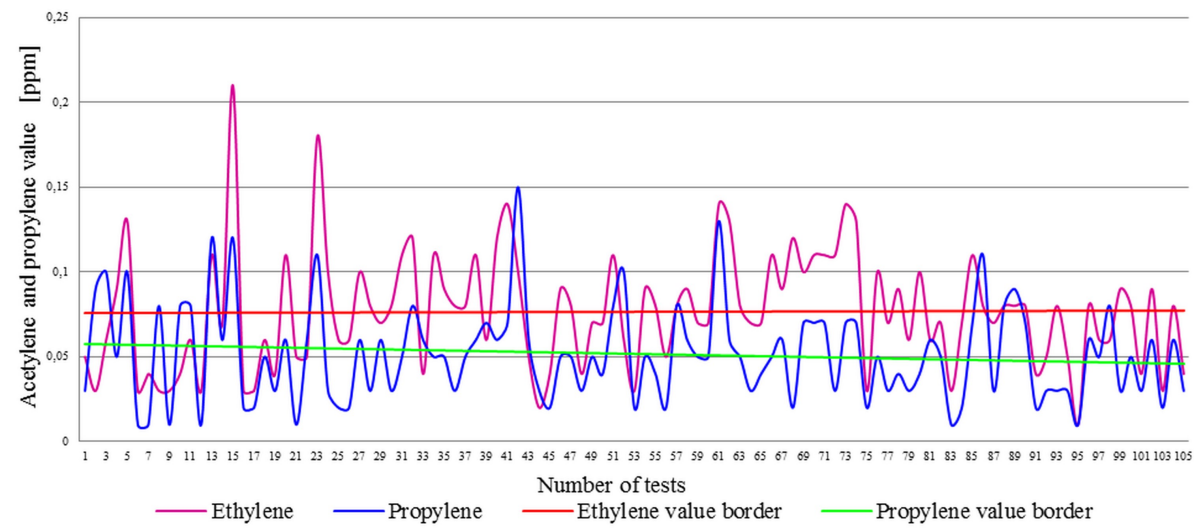

Fig. 5. Concentration of ethylene and propylene in gas samples taken from goafs of an active longwall using an integrated method to reduce the risk of endogenous fires.

The results indicate that the application of the developed method significantly reduced the threat of endogenous fires. A comprehensive approach to the problem of fires had very satisfying results. The operation and recommissioning were carried out without major disturbances.

\section{Conclusion}

The integrated method of limiting the risk of endogenous fires in hard coal mines presented in the article is a response to the growing threat of endogenous fires during underground mining exploitation. This phenomenon may create unfavourable mining conditions lead to substantial economic losses, and additionally poses a threat to the safety of employees. The effectiveness of the developed method is particularly evident in the case of exploitation of longwalls with a short incubation time. Operational progress is then crucial and without this method it would be difficult to control the threat.

It should be emphasized that the activities included the developed method were applied practically from the beginning of mining operation. Only then the probability of fire can be fully reduced. The resulting drop in the value of fire hazard indicators made it possible to safely carry out mining works in the active longwall, recommissioning operation and the final isolation of longwall workings.

The whole process involved considerable expenses, but the results proved they were well invested. It is obvious that inertisation with carbon dioxide and nitrogen, advanced regulation of aerodynamic potentials and caulking with ash-water mixtures and the administration of anti-pyrogenic foams of a particularly foamed mining inhibitor are costly and labour-intensive activities. The obtained results, however, fully compensate for them.

A very important element of the developed method is also the regular conduct of precise chromatographic analyses of the atmosphere composition in goafs. Early and precise identification of a fire hazard allows for the rapid start of integrated preventive actions enabling safe exploitation of longwalls, and then their recommissioning.

The number of isolated and recently caulked longwall regions due to the fire risk indicates that the presented method has a good chance of wide practical application. According to the authors, its application significantly improves the safety of operation by reducing the endogenous fire threat. 
The work is the result of research conducted as part of the project carried out by Polska Grupa Górnicza S.A. "Learning Organization". Its aim is to strive to actively guide the future of the company.

\section{References}

1. J. Brodny, M. Tutak, SGEM Conf. Proceed., 1:2, 299-306 (2016) DOI: 10.5593/SGEM2016/B12/S03.039

2. J. Brodny, M. Tutak, Tehnički vjesnik, 25, 271-276 (2018) doi.org/10.17559/TV20160322194812.

3. J. Brodny, M. Tutak, A. John, Mechanics, 24, 43-49 (2018)

4. J. Brodny, M. Tutak, A. John, Mechanika, 24:5, 695-702 (2018)

5. J. Brodny, M. Tutak, Journal of Applied Fluid Mechanics, 11:3, 545-553 (2018)

6. J. Brodny, M. Archives of Control Science, 29:1, 25-39 (2019)

7. J. Brodny, M. Tutak, SGEM Conf. Proceed., 1:2, 897-904 (2016) DOI : 10.5593/SGEM2016/B12/S03.115

8. D. Felka, J. Brodny, Advances in Intelligent Systems and Computing, 637, 151-160 (2017) DOI: 10.1007/978-3-319-64465-3_15

9. Raport 2017 (Górnictwo Węgla Kamiennego w Polsce, Kraków, 2018)

10. D. Szurgacz, L. Sobik, J. Brodny, Geolinks International Conference on Geosciences. Conference proceedings, 1:1, 201-209 (2019)

11. M. Tutak, J. Brodny, IOP Conf. Series: Earth Env. Sci., 95, 042025 (2017) DOI: $10.1088 / 1755-1315 / 95 / 4 / 042025$

12. M. Tutak, J. Brodny, Energies, 11:11, 3076 (2018)

13. M. Tutak, J. Brodny, E3S Web Conf., 29, 2267, (2018)

14. M. Tutak, J. Brodny, J. Env. Res. Pub. Health, 16, 1406 (2019) DOI: 10.3390/ijerph 16081406

15. M. Tutak, J. Brodny, IOP Conf. Ser.: Ear. Env. Sci., 95, 1755 (2017) DOI : $10.1088 / 1755-1315 / 95 / 4 / 042026$

16. M. Tutak, SGEM2017 Conf. Proceed., 17:43, 301-308 (2017) DOI: $10.5593 / \mathrm{sgem} 2017 \mathrm{H} / 43 / \mathrm{S} 19.038$.

17. M. Tutak, SGEM 2017 Conf. Proceed., 17:43, 53-60 (2017) DOI: $10.5593 /$ sgem $2017 \mathrm{H} / 15 / \mathrm{S} 06.007$ 